\title{
ADMISSION CONTROL ALGORITHM FOR WIRELESS COMMUNICATION NETWORKS CONSIDERING ADJUSTABLE Channel Separation
}

\author{
Chih-Hao Lin and Frank Yeong-Sung Lin \\ Department of Information Management \\ National Taiwan University \\ 50, Lane 144, Keelung Rd., Sec. 4 \\ Taipei, Taiwan, R.O.C. \\ Email: d5725001@im.ntu.edu.tw
}

\begin{abstract}
In this paper, we consider the problem of realtime distributed admission controls and a centralized static sequential homing policy for channelized wireless communication networks. To maximize spectrum efficiency, we study the effect of adjustable channel separation together considering generic channel interference on different propagation environments. For maximizing system revenue purpose, sequential homing policies can cooperate with channel assignment to prearrange channel resource more efficiency.

The emphasis of this work is to develop a centralized sequential homing algorithm and determine fixed channel allocation scheme to support realtime distributed admission control. We formulate the sequential homing problem as a combinatorial optimization problem, where the objective function is to minimize the total call blocking rate of systems. The basic solution approach is Lagrangean relaxation. In the computational experiments, we compared the proposed algorithm with the power dominant heuristic on test networks. The proposed algorithm average achieved up to $99 \%$ improvement of the total call blocking rate.
\end{abstract}

Keywords: Admission Control, Channel Assignment, Channel Separation, Sequential Homing Algorithm, and Sectorization Wireless Networks.

\section{INTRODUCTION}

In channelized cellular systems, a given radio spectrum can be divided into a set of disjoint radio channels. All channels can be used simultaneously while maintaining an acceptable received radio signal. Channel interference caused by frequency reuse is the most restraining factor on the overall system capacity in the wireless networks. The main idea behind channel assignment algorithms is to make use of radio propagation path loss characteristics and IF filter in order to minimize the carrier-to-interference ratio (CIR) and hence increase the radio spectrum reuse efficiency [2][9].

In this paper, we consider the problem of admission control for channelized wireless communication networks under the consideration of adjustable channel separation and generic channel interference. We explore the feasibility of optimizing the frequency spacing between carriers such that the combined effect of distance and IF filter to allow maximum number of channels in a given propagation environment. To accommodate different situations of smart antenna deployments, we adopt generic sectorization model to formulate irregular cell location, transmission power, and sectorization architectures. Both co-channel interference (CCI) and adjacent channel interference (ACI) are accumulated as total interference to evaluate communication quality of service $(Q \circ S)$ [2]. For maximizing system revenue purpose, sequential homing policies can cooperate with fixed channel assignment mechanism to rearrange channel resource more efficiency and support realtime distributed admission control.

We formulate the sequential homing problem as a combinatorial optimization problem, where the objective function is to minimize total blocking rate of systems subject to configuration, sequential homing, and QoS constraints. The configuration constraints require that the assigned channels for each sector be admissible. Whereas, the QoS constraints require that the call blocking probability constraint for each sector and CIR constraint received by each mobile terminal be satisfied. The basic approach to the algorithm development is Lagrangean relaxation, which has been successfully adopted to solve many famous NP-complete problems. In the computational experiments, the proposed algorithm is shown to be efficient and effective.

The remainder of this paper is organized as follows. In Section 2, the admission control problem and sequential homing problem are presented. Section 3 shows a mathematical formulation and a solution procedure for the problem. In Section 4, a primal heuristic and several computational experiment results are presented.

Proceedings of the 2002 IEEE Canadian Conference on Electrical \& Computer Engineering 0-7803-7514-9/02/\$17.00 @ 2002 IEEE 


\section{PROBLEM DESCRIPTION}

We adopt the most widely quoted macro-cell model become our propagation prediction model. The model involves dividing the prediction area into open, suburban, and urban areas. Hata's approximations [3] are described as follows [10]:

Urban areas: $L_{d B}=A+B \log R-E$

Suburban areas: $L_{d B}=A+B \log R-C$

Open areas: $L_{d B}=A+B \log R-D$

Where

$A=69.55+26.16 \log f_{c}-13.82 \log h_{b}$

$B=44.9-6.55 \log h_{b}$

$C=2\left(\log \left(f_{c} / 28\right)\right)^{2}+5.4$

$D=4.78\left(\log f_{c}\right)^{2}+18.33 \log f_{c}+40.94$

$E=3.2\left(\log \left(11.75 h_{m}\right)\right)^{2}-4.97$

(for large cities, $f_{c} \geq 300 \mathrm{MHz}$ )

$E=8.29\left(\log \left(1.54 h_{m}\right)\right)^{2}-1.1$

(for large cities, $f_{c}<300 \mathrm{MHz}$ )

$$
E=\left(1.1 \log f_{c}-0.7\right) h_{m}-\left(1.56 \log f_{c}-0.8\right)
$$

(for medium to small cities).

Notation $f_{c}$ denotes carrier frequency $(\mathrm{MHz})$, $h_{b} / h_{m}$ is BS/MT height above local terrain height (m), and $R$ is distance from BS to MT (Km). This empirical propagation prediction model can be applied in built-up quasi-smooth areas for distances between 1 and $20 \mathrm{~km}$ and for frequencies in the range of $150 \mathrm{MHz}$ to $1 \mathrm{GHz}$ (It had be extended to cover up to $100 \mathrm{~km}$ and $2 \mathrm{GHz}$ ).

In this paper, we explore the influence of adjustable channel separation on different propagation model to maximize the spectrum efficiency. If the transmitter and receiver are matched, we can use $S(f)$ to denote the filter characteristic. A ratio called as net filter discrimination (NFD) describes the discrimination of ACI over $\mathrm{CCI}$ due to channel separation and modulation spectrum shape. This NFD ratio between the received ACI and CCI can be defined as [9]

$$
N F D(\Delta f)=\int_{-\infty}^{\infty} S(f) \cdot S(f+\Delta f) d f / \int_{-\infty}^{\infty} S^{2}(f) d f .
$$

With the concept of NFD, the interferences can be formed as a function of channel separation normalized to the bit-rate. We can formulate the CIR constraint by accumulate all interferences received by each MT and ensure the receiver sensitivity of MT $t[6]$.

\subsection{Admission Control Problem}

Admission control is the acceptance or blocking of call requests. At the cell level, flow enforcement mechanisms police a source to ensure that its blocking probability does not exceed the negotiated limit [8] Admission control combined with flow enforcement (policing) can support preventive congestion control mechanism to maximize system revenue subject to the QoS constraint for each MT [7].

Channel allocation schemes can be divided into two kinds: fixed channel allocation (FCA) and dynamic channel allocation (DCA). In general, there is a trade-off between QoS, the implementation complexity of the channel allocation algorithms, and spectrum utilization efficiency. DCA strategies are less efficient than FCA under high load conditions but provide flexibility and traffic adaptability [5].

In this paper, realtime distributed admission control does not cooperate with DCA but with sequential homing based FCA mechanism. We propose a sequential homing algorithm to determine homing sequences and FCA of total system to achieve efficient of channel resources. Whenever we determine the homing sequence for each MT, admission control is source-driven so that MT initial the call setup phase and inspect the QoS feasibility of candidate homes sequentially. These admittance computations are avoided at intermediate nodes to support distributed and realtime characteristics. Another design philosophy behind the construction of the algorithm is that the speed of the decisions is more important than how close the solution is to an optimal solution [8].

\subsection{Sequential Homing Problem}

To support realtime admission control, we formulate a fixed channel resource allocation and sequential homing decision algorithm, denoted as fixed sequential homing algorithm. The architecture of target network is described by the following information and the given parameters, which are depicted in Table 1.

(1) Architectures of wireless communication networks: carrier frequency, available bandwidth, and channel separation;

(2) Configurations of base stations (BSs): location, antenna height above local terrain height, smart antenna structures, transmission power, and maximum channel configuration constraint for each BS;

(3) Characteristics of mobile terminals (MTs): antenna heights, receiver sensitivity, candidate set of homing sectors, and mean arrival rate of new traffic for each MT.

For each MT, we use Erlang-B formula to model telephony communication networks as an $\mathrm{M} / \mathrm{G} / \mathrm{m} / \mathrm{m}$ queueing system under the assumptions that overflow traffic also behaves as Poisson arrival process and average traffic load is used to estimate realtime traffic load.

We formulate the problem as an integer programming problem where the objective function is to minimize the total call blocking rate of system subject to single home 
constraints, sequential homing constraints, QoS constraints, and configuration constraints. We depict the notations of decision variables in Table 2 and the problem formulation in the following.

Table 1. Notations descriptions for given parameters.

\begin{tabular}{|c|l|}
\hline \multicolumn{2}{|c|}{ Given Parameters } \\
\hline Notation & \multicolumn{1}{|c|}{ Descriptions } \\
\hline$T$ & The set of mobile terminals \\
\hline$H_{t}$ & $\begin{array}{l}\text { The set of candidate home sectors which can } \\
\text { support of MT } t \in T\end{array}$ \\
\hline$F$ & The set of available channels \\
\hline$C$ & The set of sectors in the system \\
\hline$S$ & The set of permutation which is integer value \\
\hline$\lambda_{t}$ & $\begin{array}{l}\text { The mean arrival rate of new traffic for each } \\
\text { MT } t \in T\end{array}$ \\
\hline$\gamma$ & Threshold of acceptable CDR \\
\hline$g_{j}$ & Upper bound of aggregate traffic for Sector $j$ \\
\hline$N_{j}$ & $\begin{array}{l}\text { Upper bound of assigned channels for Sector } \\
j \in C\end{array}$ \\
\hline$\delta_{v}$ & $\begin{array}{l}\text { Indicator function which is 1 if Sector } j \\
\text { belongs to homing policy } h \in H, \text { and } 0 \\
\text { otherwise }\end{array}$ \\
\hline$a_{v}$ & $\begin{array}{l}\text { Received power level of MT } t \in T \text { from } \\
\text { the downlink signal of Sector } j \in C\end{array}$ \\
\hline$d_{j}$ & $\begin{array}{l}\text { Blocking probability function of Sector } j \\
\text { which is a function of traffic demand and } \\
\text { available channels. }\end{array}$ \\
\hline$\left.\Delta_{i}\right)$ & $\begin{array}{l}\text { NFD ratio which is formed as a function of } \\
\text { the channel separation (kHz) normalized to } \\
\text { the bit-rate (bps) }\end{array}$ \\
\hline
\end{tabular}

Table 2. Notations descriptions for decision variables.

\begin{tabular}{|c|l|}
\hline Decision Variables \\
\hline Notation & \multicolumn{1}{|c|}{ Descriptions } \\
\hline$g_{j}$ & $\begin{array}{l}\text { Aggregate flow on Sector } j \in C \text { (in Er- } \\
\text { langs) }\end{array}$ \\
\hline$y_{s}$ & $\begin{array}{l}\text { Channel assignment decision variable which } \\
\text { is } 1 \text { if Channel } i \in F \text { is assigned to Sector } \\
j \in C \text { and 0 otherwise }\end{array}$ \\
\hline$x_{h s}$ & $\begin{array}{l}\text { Homing decision variable which is } 1 \text { if hom- } \\
\text { ing decision } h \in H_{t} \text { of } \mathrm{MT} \quad t \in T \quad \text { is } \\
\text { selected as the } s \text { th candidate path and } 0 \\
\text { otherwise }\end{array}$ \\
\hline$B_{s}$ & $\begin{array}{l}\text { Call blocking probability for the sth candi- } \\
\text { date homing policy for } t \text { which belongs to } \\
\text { discrete set } B_{u} \in K_{u}=\left\{0,0.01,0.02, \ldots, \bar{B}_{b}\right\}\end{array}$ \\
\hline$d_{v}$ & $\begin{array}{l}\text { Blocking probability of Sector } j \text { which is } \\
\text { referenced by } M T \quad t \in T\end{array}$ \\
\hline
\end{tabular}

Objective function (IP):

$$
Z_{I P}=\min \sum_{t \in T}\left(\lambda_{t} \prod_{s e S} B_{t}\right)
$$

subject to:

$$
\begin{aligned}
& \sum_{k \in B_{t}} x_{k s} \sum_{j \in C} \delta_{v} d_{v}=B_{v} \\
& \forall t \in T, s \in S \\
& d_{j}\left(\sum_{i \in F} y_{j}, g_{j}\right)=d_{v} \\
& \forall t \in T, j \in C \\
& \sum_{k \in T} \sum_{k \in H_{i}}\left(\lambda_{t} \delta_{k j} \sum_{s \in S}\left(x_{k} \prod_{k=1}^{s-1} B_{d k}\right)\right)=g_{j} \\
& \forall j \in C \\
& \sum_{i \in B_{t}} \delta_{k j} \sum_{j \in C} \sum_{j \in F} \frac{a_{b^{\prime}}}{a_{i j}} y_{j l} \theta\left(\left|i-i^{\prime}\right|\right) \leq G_{j}+\left(\frac{1}{\gamma}+1-G_{j}\right) y_{j} \\
& \forall i \in F, j \in C, t \in T \\
& \sum_{i \in F} y_{j i} \leq N_{j} \\
& \forall j \in C \\
& \sum_{k \in B_{t}} x_{k s}=1 \\
& \forall t \in T, s \in S \\
& \sum_{s e S} x_{k s}=1 \\
& \forall t \in T, h \in H \\
& x_{k s}=0 \text { or } 1 \\
& y_{A}=0 \text { or } 1 \\
& \forall t \in T, h \in H_{t}, s \in S \\
& \forall j \in C, i \in F \\
& 0 \leq B_{b} \leq \bar{B}_{s} \\
& 0 \leq d_{b j} \leq \bar{d}_{v} \\
& \forall t \in T, s \in S, B_{b} \in K_{u} \\
& \forall t \in T, j \in C \\
& 0 \leq g_{j} \leq \bar{g}_{j} \\
& \forall j \in C .
\end{aligned}
$$

The objective is to minimize the call blocking rate of total system. Constraint (1) is the call blocking probability of MT $t$ on the permutation $s$. Constraint (2) decomposes the call blocking probability of Sector $j$ by introducing one additional notation $d_{v}$. Constraint (3) calculates the aggregate traffic for Sector $j \in C$. Constraint (4) ensure CIR requirement for mobile terminals, which are homed to Sector $j \in C$. Constraint (5) enforces configuration constraint for Sector $j \in C$. Constraint (6) enforce only one candidate homed BS can be selected for each MT $t$ on each permutation $s$. Constraint (7) enforces the number of candidate path is equal to the number of homing decisions. Constraints (8) and (9) enforce the integer property of the decision variables $x_{k s}$ and $y_{f}$. Constraint (10) limits the feasible discrete region of path blocking probability. Constraints (11) and (12) enforce the feasible regions of call blocking probability and aggregate traffic for each Sector $j$.

\section{SOLUTION PROCEDURE}

The above fixed sequential homing problem is NP-complete, and we do not expect to develop an opti- 
mal algorithm for large-scale problems. Instead, an efficient Lagrangean-based algorithm is developed.

\subsection{Lagrangean Relaxation}

By using Lagrangean relaxation method [1], we relax three complicate constraints. Two of them are integer programming problems, which are Constraints (2) and (4), and another one is signomial problem, that is Constraint (3). These constraints must be multiplied by Lagrangean multipliers and added to the objective function. After dualizing these complicating constraints, we can construct the following Lagrangean relaxation problem.

Objective function (LR):

$$
\begin{aligned}
& Z_{D}\left(\mu_{v}^{1}, \mu_{j}^{2}, \mu_{j t}^{3}\right)=\min \sum_{k \in T}\left(\lambda_{i} \prod_{s \in S} B_{b}\right) \\
& +\sum_{i \in T} \sum_{j \in C} \mu_{v}^{1}\left(d_{j}\left(\sum_{i \in F} y_{j}, g_{j}\right)-d_{v}\right) \\
& +\sum_{j \in C} \mu_{j}^{2}\left(\sum_{j \in T} \sum_{k \in B_{t}}\left(\lambda_{t} \delta_{k j} \sum_{j \in S}\left(x_{k j} \prod_{k=1}^{s-1} B_{t}\right)\right)-g_{j}\right) \\
& +\sum_{j \in C} \sum_{i \in F} \sum_{k \in T} \mu_{A^{\prime}}^{3}\left(\sum_{h \in H_{t}} \delta_{w} \sum_{j \in C} \sum_{j \in F}\left(\frac{a_{g^{\prime}}}{a_{y}} y_{j \gamma^{\prime}} \theta\left(\left|i-i^{\prime}\right|\right)\right)\right. \\
& \left.-G_{j}-\left(\frac{1}{\gamma}+1-G_{j}\right) y_{n}\right)
\end{aligned}
$$

subject to: (1), (5), (6), (7), (8), (9), (10), (11), and (12).

In this formulation, all of $\mu_{y}^{1}, \mu_{j}^{2}, \mu_{k t}^{3}$ are Lagrange multipliers but only $\mu_{\mu t}^{3}$ must be positive. To solve this problem, we can decompose (LR) into the following two independent and solvable optimization subproblems.

Subproblem 1 (related with decision variables $B_{t}$, $x_{k x}$, and $d_{v}$ )

$$
\begin{aligned}
& \text { Objective function: } \\
& Z_{\text {SUB1 }}=\min \sum_{v \in T}\left(\lambda_{t} \prod_{J \in S} B_{v}\right)-\sum_{v \in T} \sum_{j \in C} \mu_{v}^{1} d_{v}
\end{aligned}
$$

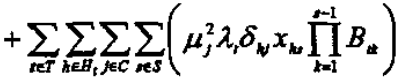

$$
\begin{aligned}
& \text { subject to: (1), (6), (7), (8), (10), and (11). }
\end{aligned}
$$

We can decompose this problem into $|T|$ subproblems. Each subproblem solves the following problem.

$$
\begin{aligned}
& Z_{S U B_{t}}=\min \lambda_{t} \prod_{v e S} B_{v}-\sum_{j \in C} \mu_{v}^{1} d_{v} \\
& +\sum_{k \in B_{t}} \sum_{j \in C} \sum_{s \in S}\left(\mu_{j}^{2} \lambda_{t} \delta_{\psi} x_{t s} \prod_{t=1}^{s-1} B_{t k}\right) \forall t \in T
\end{aligned}
$$

As the property of decision variable $x_{k s}$ and given parameter $\delta_{\psi}$, we can exhaustively search for all possi- ble combination value of $x_{k s} \times \delta_{k j}$ to calculate minimum objective value for subproblem 1 .

Subproblem 2 (related with decision variables $y_{f}$ and $g_{j}$ )

$$
\begin{aligned}
& Z_{s v B 2}=\min \sum_{\text {ter }} \sum_{j \in C} \mu_{v}^{1} d,\left(\sum_{j \in F} y_{j}, g_{j}\right)-\sum_{j \in C} \mu_{j}^{2} g_{j} \\
& +\sum_{j \in C} \sum_{i \in F} \sum_{k \in T} \mu_{j i}^{3}\left(\sum_{n \in H_{t}} \delta_{w} \sum_{j \in C} \sum_{i \in \mathcal{F}} \frac{a_{v}}{a_{v}} y_{j i} \theta\left(\left|i-i^{\prime}\right|\right)\right. \\
& \left.-G_{j}-\left(\frac{1}{\gamma}+1-G_{j}\right) y_{j}\right)
\end{aligned}
$$

subject to: (5), (9), and (12).

We can decompose this problem into $|C|$ subproblems. Each subproblem solves the following problem.

$$
\begin{aligned}
& Z_{s U s 2_{c}}=\min \sum_{t \in T} \mu_{i}^{1} d_{j}\left(\sum_{i \in F} y_{j i}, g_{j}\right)-\mu_{j}^{2} g_{j}-\sum_{j \in F} \sum_{t \in T} \mu_{f i t}^{3} G_{j}
\end{aligned}
$$

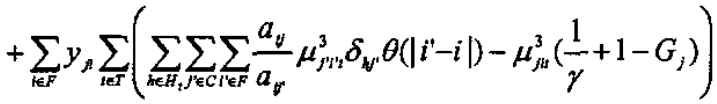

$\forall j \in C$.

According to the weak Lagrangean duality theorem, for any $\mu_{j t}^{3} \geq 0$ and $\mu_{\theta}^{1}, \mu_{f}^{2}$, the objective value of $Z_{D}\left(\mu_{v}^{1}, \mu_{j}^{2}, \mu_{j i}^{3}\right)$ is a lower bound of $Z_{I P}$. The following dual problem (D) is then constructed to calculate the tightest lower bound.

$$
Z_{D}=\max _{\mu_{j s}^{3} \geq 0} Z_{D}\left(\mu_{t i}^{1}, \mu_{j}^{2}, \mu_{j i t}^{3}\right) \text {. }
$$

There are several methods for solving the dual problem (D), of which the subgradient method is the most popular and is employed here [4]. In iteration $k$ of the subgradient optimization procedure, the multiplier vector $\pi$ is updated by $\pi^{k+1}=\pi^{k}+t^{k} g^{k}$. The step size $t^{*}$ is determined by $t^{k}=\delta\left(Z_{p^{p}}^{k}-Z_{D}\left(\pi_{k}\right)\right) /\left\|g^{k}\right\|^{2}$, where $Z_{P p}^{h}$ is the primal objective function value for a heuristic solution. It is an upper bound on $Z_{\mathrm{IP}}$.

\subsection{Getting Primal Feasible Solution}

When we use Lagrangean relaxation and subgradient method as our solution approaches to solve the problems, we not only get a theoretical lower bound of primal feasible solution, but also get some hints when solving dual problem iteratively. More precisely, when solving the dual problem iteratively, a Lagrangean relaxation problem is solved. If the decision variables are feasible and satisfy all of the constraints in the primal problem, then a primal feasible solution is found. Otherwise, modification on such infeasible primal solutions can be made to obtain primal feasible solutions. For getting primal fea- 
sible solutions purpose, we propose a homing dominant primal algorithm, denoted by Algorithm $A$. In Algorithm $A$, we use the permutation results as the dominant decision and then allocate available channels by load-balance approach.

[Algorithm $A$ ]

Step 1. Initialize permutation $s=0$ to denote the first homing situation for all of MTs.

Step 2. For each sector $j \in C$, applying the sequential homing policy, referred to the decision variable $x_{k s}$ of MT $t$ from Lagrangean dual problem, to pre-calculate the aggregate traffic under permutation $s$ situation by Constraint (3): $\sum_{k \in T} \sum_{k \in H_{i}}\left(\lambda_{t} \delta_{w} \sum_{s \in S}\left(x_{k v} \prod_{k=1}^{s-1} B_{t}\right)\right)=g_{j}$.

Step 3. For channel assignment purpose, we calculate the blocking rate reduction level for each sector $j \in C$ and then sort sector order by their contribution of reducing objective value if assigning one additional channel to sector.

Step 4. Using the comments of Lagrangean dual problem to sort channel order for Sector $j$. The comments are calculated from the coefficient of channel assignment decision variables $y_{j}$

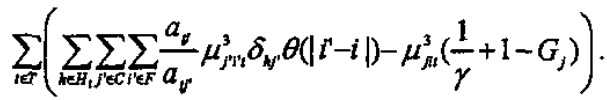

Step 5. Assign the lowest coefficient channel $i$ to the greatest objective reduction sector $j$ and then check the feasibility of this assignment. We must ensure the configuration constraints for Sector $j$ (Constraint (5)) and the QoS constraints for each MT that is homed to Sector $j$ (Constraint (4)).

Step 6. If the QoS constraints are feasible, assign this Channel $i$ to Sector $j$, change the system status, resort sector order, and go to Step 3. Otherwise, retry to assign next channel for Sector $j$ by go to Step 5. If the configuration constraints or blocking probability constraints violate, go to Step 7 to apply drop-and-add approach to modify sequential homing policy.

Step 7. For tuning infeasible solution purpose, we sort all of sectors by its residue capacity in decreasing order to construct modify sector order. This sector order reveals the sector modifiability to reroute more traffic from infeasible sectors.

Step 8. Reroute the traffic load of multiple candidate-home MT from the most infeasible sector to the greatest modifiability sector. Check the feasibility of both modified sectors and modify the sequential homing decision.
Step 9. If the most infeasible sector still infeasible, go to Step 8 to reroute more traffic. If any sector still infeasible, go to Step 7 for further tuning.

Step 10. If all of permutation cases have processed, calculate the objective value and keep the best feasible solution, Otherwise, set $s=s+1$ and go to Step 2 for processing next permutation phase.

\section{COMPUTATIONAL EXPERIMENTS}

In this section, we randomly generate a sectorization wireless network topology as our experiment environment. In this topology, there are 5 BSs constructed by 15 smart antenna to service $20 \mathrm{MT}$ clusters under the GSM-like situation that frequency $\left(f_{c}\right)$ is on $900 \mathrm{MHz}$, bandwidth $(W)$ is $12.5 \mathrm{MHz}, \mathrm{CIR}(\gamma)$ is $9 \mathrm{~dB}$, average MT height $\left(h_{m}\right)$ is between $1 \mathrm{~m}$ to $10 \mathrm{~m}$, average BS height $\left(h_{b}\right)$ is between $30 \mathrm{~m}$ to $200 \mathrm{~m}$. For comparison purpose, we also develop a primal heuristic as follows.

\subsection{Primal Heuristic}

For developing a primal heuristic, we adopt the most traditional homing policy which home each MT prioritizes to the greatest received downlink power sector. We denote this power dominant homing policy as Heuristic $H$ and describe in the following. In Heuristic $H$, the homing permutation is in decreasing order of received power. The sector order for assigning channel is the same with Algorithm $A$ by sorting the contribution of reducing objective value in decreasing order. For simplicity purpose, we assign channel just by the order of channel identifiers. The detail description of this primal heuristic is described in the following.

[Heuristic $H$ ]

Step 1. For each MT $t \in T$, following received power dominant rule to become the homing sequence of each MT. Initialize $s=0$ and begin to process the first homing phase.

Step 2. For each sector $j \in C$, calculate the aggregate traffic on each permutation situation $s$.

Step 3. Sort sector order by blocking rate reduction level of each sector in decreasing order.

Step 4. Using the channel identifier as the channel order, check sequentially the feasibility of channel assignment policy for the greatest objective reduction sector $j$.

Step 5. If the QoS constraints are feasible, assign this Channel $i$ to Sector $j$, change the system status, resort sector order, and go to Step 3. Otherwise, retry to assign next channel for Sector $j$ by go to Step 4. If the configuration constraints or blocking probability constraints violate, rehome the 
MT to next candidate home sector.

Step 6. If all of permutation cases have processed, calculate the objective value and keep the best feasible solution. Otherwise, set $s=s+1$ and go to Step 2 for processing next permutation phase.

\subsection{Experiment Results}

After several computational experiments, we list all of the experiment results together in Table 3. For exploring the effect of adjustable channel separation, the experiments are performed the network topology on different propagation environment to evaluate the effect of adjustable channel separation. In these cases, we can find that different propagation environments bave different niche channel separation. In this GSM-like test environment, the performance of $100 \mathrm{kHz}$ channel separation is better than that of the standard GSM system, which channel separation, is $200 \mathrm{kHz}$.

In the second experiment, we compare the computational results of Algorithm $A$ and Heuristic $H$ to evaluate the effective of the proposed algorithm. In these experiments, we can observe that as the traffic load increasing, Algorithm $A$ can achieve feasible solution but Heuristic $H$ cannot. Furthermore, Algorithm $A$ achieved average up to $99 \%$ improvement of the total call blocking rate.

\section{CONCLUSION}

In this paper, we consider the problem of realtime distributed admission controls for channelized wireless communication networks. Instead of adopting DCA policy, we develop a centralized sequential homing algorithm cooperate with fixed channel assignment to maximize spectrum efficiency. Further, we study the effect of adjustable channel separation together considering generic channel interference on different propagation environments.

We formulate the sequential homing problem as a combinatorial optimization problem and use Lagrangean relaxation as our solution approach. In the computational experiments, the proposed channel separation is better than GSM system. We also compared the proposed algorithm with the power dominant heuristic on test networks. The proposed algorithm achieved average up to $99 \%$ improvement of the total call blocking rate.

\section{References}

[1] M.L. Fisher, "The Lagrangian relaxation method for solving integer programming problems," Management Science, vol. 27, pp. 1-18, 1981 .

[2] S. Golestaneh, H.M. Hafez, and S.A. Mahmoud, "The effect of adjacent channel interference on the capacity of FDMA cellular systems," IEEE Transactions on Vehicular Technology, vol. 43, no. 4, 1994.
[3] M. Hata, "Empirical formula for propagation loss in land mobile radio services," IEEE Transactions on Vehicular Technology, vol. 29, pp. 317-325, 1980.

[4] M. Held, P. Wolfe, and H. D. Crowder, "Validation of subgradient optimization", Math. Programming, vol. 6, pp. 62-88, 1974.

[5] I. Katzela and M. Naghshineh, "Channel assignment schemes for cellular mobile telecommunication systems: a comprehensive survey," IEEE Personal Communications, vol. 3, no. 3, pp. 10-31, June 1996.

[6] C-H. Lin and F. Y-S. Lin, "Channel augmentation algorithm for wireless networks considering generic sectorization and channel interference," Proc. IEEE $M W C N$, Brazil, 2001.

[7] F. Y-S. Lin and C-T. Chen, "Admission control and routing algorithms for networks supporting the permanent virtual connection (PVC) service," Proc. ISCOM, 1997.

[8] F. Y-S. Lin and J.R. Yee, "A real-time routing and admission control algorithm for ATM networks," Proc. IEEE INFOCOM, 1993.

[9] P. Malm and T. Maseng, "Adjacent channel separation in mobile cellular systems," Proc. IEEE VTC, vol. 2, 1997.

[10]S.R. Saunders, Antennas and Propagation for Wireless Communication systems, John Wiley \& Sons, 1999.

Table 3. Experiment results for Algorithm $A$ and Heuristic $H$ upon several test networks.

\begin{tabular}{|c|c|c|c|c|c|c|}
\hline Case & Areas & $\Delta i$ & $\lambda_{i}$ & Algorithm $A$ & Heuristic $H$ & $\begin{array}{c}\text { Improve- } \\
\text { ment }\end{array}$ \\
\hline 1 & open & 50 & 2 & $8.4781 \mathrm{e}-10$ & $1.1600 \mathrm{e}-04$ & $99.9 \%$ \\
\hline 2 & open & 100 & 2 & $5.8552 \mathrm{e}-19$ & $8.2101 \mathrm{e}-04$ & $100 \%$ \\
\hline 3 & open & 200 & 2 & $2.6054 \mathrm{e}-13$ & $1.0501 \mathrm{e}-02$ & $100 \%$ \\
\hline 4 & open & 300 & 2 & $2.6515 \mathrm{e}-04$ & $1.7376 \mathrm{e}-01$ & $99.8 \%$ \\
\hline 5 & open & 50 & 5 & $6.6102 \mathrm{e}-03$ & $9.0580 \mathrm{e}-01$ & $99.2 \%$ \\
\hline 6 & open & 100 & 5 & $3.2364 \mathrm{e}-09$ & $9.6843 \mathrm{e}-01$ & $99.9 \%$ \\
\hline 7 & open & 200 & 5 & $2.0477 \mathrm{e}-03$ & $3.0707 \mathrm{e}+00$ & $99.9 \%$ \\
\hline 8 & open & 300 & 5 & $5.0930 \mathrm{e}+00$ & N/A & - \\
\hline 9 & open & 100 & 10 & $1.7254 \mathrm{e}-07$ & N/A & - \\
\hline 10 & open & 200 & 10 & $5.4591 \mathrm{e}+00$ & N/A & - \\
\hline 11 & open & 300 & 10 & N/A & N/A & - \\
\hline 12 & urban & 50 & 2 & $1.1209 \mathrm{e}-13$ & $9.9436 \mathrm{e}-05$ & $100 \%$ \\
\hline 13 & urban & 100 & 2 & $7.0688 \mathrm{e}-26$ & $2.5741 \mathrm{e}-04$ & $100 \%$ \\
\hline 14 & urban & 200 & 2 & $7.6380 \mathrm{e}-05$ & $2.5839 \mathrm{e}-02$ & $99.7 \%$ \\
\hline 15 & urban & 300 & 2 & $3.8196 \mathrm{e}-04$ & $6.3651 \mathrm{e}-01$ & $99.9 \%$ \\
\hline 16 & urban & 50 & 5 & $1.6912 \mathrm{e}-02$ & $2.9121 \mathrm{e}+00$ & $99 \%$ \\
\hline 17 & urban & 100 & 5 & $3.5969 \mathrm{e}-06$ & $2.9843 \mathrm{e}+00$ & $99.9 \%$ \\
\hline 18 & urban & 200 & 5 & $9.7828 \mathrm{e}-01$ & N/A & -- \\
\hline 19 & urban & 300 & 5 & $1.5764 \mathrm{e}+01$ & N/A & - \\
\hline
\end{tabular}

